Zbl Arbeitsmed 2022 $\cdot 72: 68-77$ https://doi.org/10.1007/s40664-021-00453-7 Eingegangen: 18. Juni 2021

Angenommen: 3. November 2021

Online publiziert: 17. Januar 2022

(c) Der/die Autor(en) 2022

Holger Hoffmann · Imke Pitz · Björn Adomssent · Christoph Russmann

Fakultät für Ingenieurwissenschaften und Gesundheit, HAWK Hildesheim/Holzminden/Göttingen, Göttingen, Deutschland

\title{
Assoziation, Erwartungen und Barrieren eines Exoskeletteinsatzes in kleinen mittelständischen Unternehmen
}

\section{Theoretischer Hintergrund und Fragestellung}

Im produzierenden Gewerbe und der Logistik nehmen arbeitsbedingte MSE $\mathrm{zu}$ und verursachen eine hohe Zahl an Krankheitstagen sowie körperliche Einschränkungen mit erheblichen wirtschaftlichen Folgen. MSE sind in Deutschland die Ursache für bis zu 27\% aller Produktionsausfälle durch krankheitsbedingte Abwesenheit [3, 34]. 2015 litten $52 \%$ der Arbeitnehmer in Deutschland in den letzten 12 Monaten unter MSE. Als ökonomische Folgen resultieren geminderte Produktivität und höhere Sozialausgaben. Die damit verursachten Kosten belasteten das Gesundheitssystem und die Volkswirtschaft Deutschlands allein in 2016 mit 17,2 Mrd. € [13]. MSE werden durch physisch belastende Tätigkeiten, in denen durch Muskelkraft schwere Lasten bewegt, gehoben oder getragen werden, begünstigt. Erscheinen diese unvermeidbar, sind Unternehmen gesetzlich verpflichtet, technische, organisatorische und Schulungsmaßnahmen anzubieten und, wo nötig, personenbezogene Schutzmaßnahmen zu treffen sowie Schutzausrüstung einzusetzen $[6,7]$. Aktive und passive Exoskelette können den Körper mechanisch unterstützen $[22,28]$ und fallen durch vielseitigen Einsatzzweck unter unterschiedliche dieser Maßnahmenkategorien und den damit einhergehenden sicherheitstechnischen Regularien [11]. Aktive Exoskelette unterscheiden sich von passiven insofern, dass diese einen elektrischen oder pneumatischen Antrieb vorweisen und dadurch größer und schwerer sind, aber generell mehr Unterstützung bieten können als passive. Passive Exoskelette sind durch ihre rein mechanisch, über Federwirkungen oder Expander, generierte Unterstützung leichter und kostengünstiger [36]. Speziell beim Heben, Tragen und Umlagern von Lasten und bei statischen Körperhaltungen wie Überschulterarbeiten können Exoskelette wirksam unterstützen [32]. Durch die Unterstützung reduziert sich die subjektive Belastung der Nutzer, und die Wahrscheinlichkeit, an einer arbeitsbedingten MSE zu erkranken, könnte minimiert werden $[15,17]$. Auch objektive Entlastungen konnten nachgewiesen werden. Verschiedene Studien gehen davon aus, dass die muskuläre Belastung bei Belastungsspitzen innerhalb von Aktivitäten durch das Tragen von passiven, den Rücken unterstützenden, Exoskeletten um 10 bis $40 \%$ und bei statischen Haltungen um bis zu $57 \%$ reduziert werden kann [5, 16, 21, 22, 33]. Außerdem wurde eine Verringerung der wahrgenommenen Anstrengung sowie eine Verlängerung der Ausdauerzeiten in statischen Vorwärtsbeugehaltungen festgestellt $[1,5,33]$. Studien mit passiven Schulterexoskeletten haben gezeigt, dass die Aktivierung der Schultermuskulatur für die agonistische Muskulatur bei Überschulterarbeiten um 16 bis $73 \%$ sinkt und auch die wahrgenommene Anstrengung und der Diskomfort reduziert werden $[10,19,33]$. So können Unternehmen die MSE-bedingten Kosten 


\begin{tabular}{|c|c|}
\hline Branche & Position \\
\hline Logistik & Betriebsleitung Operation \\
\hline Lagertechnik & Geschäftsführung \\
\hline Stahlhochbau & Geschäftsführung \\
\hline Baubranche & Mitarbeiter*in Marketing \\
\hline $\begin{array}{l}\text { Bauneben- } \\
\text { gewerbe }\end{array}$ & $\begin{array}{l}\text { Business Development } \\
\text { Manager*in }\end{array}$ \\
\hline $\begin{array}{l}\text { Produzierendes } \\
\text { Gewerbe }\end{array}$ & $\begin{array}{l}\text { Fachkraft für Arbeitssicher- } \\
\text { heit }\end{array}$ \\
\hline
\end{tabular}

senken, die Produktivität steigern und eine konstant hohe Qualität der Arbeit ermöglichen [10, 14, 35]. Exoskelette werden in großen Betrieben der herstellenden Industrie bereits angewendet, um muskuloskeletale Belastungen der Arbeitnehmer zu reduzieren [17, 23, 37]. Allerdings unterliegt die Ausbreitung und Akzeptanz von Exoskeletten in den Betrieben vielschichtigen Faktoren [22, 26]. So bilden ein geringer Tragekomfort, die teilweise noch einseitige Anwendbarkeit am Arbeitsplatz und eine niedrige Akzeptanz ein entscheidendes Hindernis für die Ausbreitung von Exoskeletten [5, $17,22]$.

Kim et al. [20] untersuchten die Sichtweise der US-amerikanischen Bauindustrie (Baugewerbe) $\mathrm{zu}$ dem Einsatz und Nutzen von Exoskeletten als einen ersten Schritt zur Implementierung. Es wurden die Unsicherheiten über den Gebrauchswert, die Kosten, den Nutzen und die Sicherheit der Arbeiter bei Benutzung, aber auch die Hoffnung auf gesteigerte Produktivität, verminderte Gesundheitsgefährdung sowie gesteigerte Motivation deutlich [20, 26]. Exoskelette sollen auch im Rahmen des Projektes Exskallerate in KMU der NSR eingeführt werden, und als erster Schritt einer erfolgreichen Implementierung sollen Informationen über die Erwartungen und mögliche Barrieren dieser Zielgruppe generiert werden. Um Aussagen über Assoziationen zu Exoskeletten im Rahmen der Akzeptanz für den Einsatz dieser bei KMU in der NSR zu erhalten, wurden daher im Zeitraum Dezember 2020 bis Januar 2021 Ansprechpartner aus sechs KMU interviewt. Aus diesen Ergebnissen soll ein erster Überblick über die Barrieren und Erwartun- gen eines Exoskeletteinsatzes geschaffen werden.

\section{Methode}

Es wurden teilstrukturierte Interviews geführt. Über ein RegionalmanagementUnternehmen wurden aus der Region Nordhessen und Südniedersachsen Unternehmen aus der Bau- und Logistikbranche kontaktiert und nach Rückmeldung zur Projektteilnahme eingeladen. Mit einem Teil dieser Unternehmen sollten teilstrukturierte Interviews geführt werden. Von allen Teilnehmenden lagen zu Beginn der Interviews Einwilligungs- und Datenschutzerklärung von Ansprechpartner*innen der Unternehmen vor. Es wurden Fragen für ein teilstrukturiertes Leitfadeninterview entwickelt. Die Fragen wurden so konzipiert, dass Antworten zum Wissensstand, zu Assoziationen über Exoskelette und mögliche Barrieren für die Implementierung von Exoskeletten gegeben werden konnten. Nach einer Literatursichtung zu möglichen Antworten auf die genannten Fragen wurden Antwortkategorien gebildet und die Fragen gebildet. Die Ergebnisse von Kim et al. [20] halfen unter anderem (z.B. [2, 12, 29]) bei der Kategorien- und Fragenentwicklung. Die Befragungen fanden durch einen Mitarbeiter des RegionalmanagementUnternehmens unter Verwendung einer Software für Videokonferenzen statt. Die Transkription der Audiodateien erfolgte durch eine wissenschaftliche Mitarbeiterin. Im Anschluss wurden die Transkripte von zwei Personen unabhängig voneinander analysiert und ausgewertet. Die inhaltliche Analyse fand nach dem von Mayring beschriebenen Verfahren zur Auswertung von teilstrukturierten Interviews statt [24]. Es wurden Generalisierungen und Reduktionen der Antworten durchgeführt. Diese wurden dann den bestehenden Kategorien zugeordnet. Bei Bedarf wurden neue Kategorien und Subkategorien gebildet. In - Tab. 2 sind alle Fragen des Leitfadeninterviews aufgelistet sowie alle Kategorien aufgezeigt. Es werden auch Beispiele für die Zuordnung deutlich gemacht. Durch eine Regionalmanagement-Gesellschaft wurde zunächst eine
Liste der KMU nach den Projektvorgaben erstellt. Diese Unternehmen wurden $\mathrm{zu}$ einem Workshop eingeladen. Im Rahmen dieses Workshops sollten die Interviews durchgeführt werden. Aufgrund der COVID-19-Pandemie konnte dieser nicht stattfinden. Die 18 interessierten Unternehmensvertreter ${ }^{\star}$ innen wurden daraufhin per E-Mail angefragt, ob sie bereit sind, an einem Interview teilzunehmen. Auf Basis der Anfrage haben sich sechs Betriebe zu einem Interview bereiterklärt. Jedes der sechs Unternehmen hat eine ${ }^{\star} n$ Ansprechpartner*in zur Verfügung gestellt, der ${ }^{\star}$ die die Interviewfragen stellvertretend beantwortet hat. Die Positionen dieser Ansprechpartner ${ }^{\star}$ innen im jeweiligen Unternehmen und die Branche des Unternehmens ist - Tab. 1 zu entnehmen. Im Folgenden wird von den Antworten der Unternehmen gesprochen; hier ist zu beachten, dass es sich jeweils um die Antworten der stellvertretenden Ansprechpartner handelt.

Im nachfolgenden Abschnitt der Ergebnisse werden diese Antworten dargestellt.

\section{Ergebnisse}

Die teilnehmenden Unternehmen kommen aus den Branchen: Logistik, Lagertechnik, Stahlhochbau und Bau sowie dem Baunebengewerbe und dem allgemein produzierenden Gewerbe (- Tab. 1). In allen befragten Unternehmen werden Tätigkeiten ausgeführt, die unter die Lastenhandhabungsverordnung [6] fallen und Tätigkeiten, für die nach dem Arbeitsschutzgesetz [7] geeignete, schützende Maßnahmen zu treffen sind. Erfahrungen mit Exoskeletten im beruflichen Kontext hatten drei der Befragten. Die Erfahrung reicht von Vorführungen eines Herstellers, über den rehabilitativen Kontakt bis hin zum Einsatz eines aktiven Exoskeletts im Unternehmen. Die übrigen Befragten haben keinen Kontakt mit Exoskeletten gehabt, waren jedoch teilweise bereits an Projekten interessiert (Frage 4 in - Tab. 2).

Alle befragten Unternehmen berichten von Handhabungen schwerer Materialien, die das Tragen und Heben mit und 
Zbl Arbeitsmed 2022 · 72:68-77 https://doi.org/10.1007/s40664-021-00453-7

(c) Der/die Autor(en) 2022

H. Hoffmann · I. Pitz · B. Adomssent · C. Russmann

\section{Assoziation, Erwartungen und Barrieren eines Exoskeletteinsatzes in kleinen mittelständischen} Unternehmen

\section{Zusammenfassung}

Hintergrund. Arbeitsbedingte MuskelSkelett-Erkrankungen (MSE) führen in der herstellenden Industrie zu Krankheitstagen und haben erhebliche wirtschaftliche Folgen für die Unternehmen und die Volkswirtschaft. Exoskelette können den Körper im Umgang mit schwerer Last oder in Zwangshaltungen unterstützen. Besonders in großen Unternehmen der Automobilindustrie werden Exoskelette pilotiert. In kleinen und mittelständischen Unternehmen (KMU) werden Exoskelette bisher jedoch wenig eingesetzt, und ihre Anwendung dort wurde wissenschaftlich bisher kaum untersucht. Ziel dieser Arbeit war es, die Barrieren der Exoskelett-Implementierung und die Erwartungen an deren Einsatz im produzierenden Gewerbe zu ermitteln. Methode. In sechs produzierenden Unternehmen wurden teilstrukturierte Leitfadeninterviews durchgeführt und analysiert.

Ergebnisse. In den Unternehmen werden vielfältige Tätigkeiten bis an die Belastungsgrenze ausgeführt. Allgemein erwartet man durch die Anwendung von Exoskeletten Arbeitserleichterungen sowie wirtschaftliche Vorteile. Bedenken bestehen hinsichtlich des Einsatzes aufgrund des Kostenfaktors, eines ungewissen Nutzens und mangelnden Tragekomforts. Insbesondere werden mangelnde Kenntnisse über den Effekt eines Exoskeletts deutlich.
Fazit. Die vorgestellten Interviewergebnisse sind ein Schritt im interdisziplinären Prozess der Weiterentwicklung und Implementierung von Exoskeletten in der herstellenden Industrie. Bedenken und Unwissenheit potenzieller Unternehmen und Anwender müssen adressiert werden, auch um eine hohe Nutzerakzeptanz zu schaffen. Folgestudien, die die Ermittlung des Bedarfs mit einer besseren Trennschärfe erheben, könnten weitere Erkenntnisse liefern.

Schlüsselwörter

Industrielles Exoskelett · Arbeitsbedingte muskuloskeletale Erkrankungen · Endnutzer . Herstellende Industrie · Logistik

\section{Association, expectations and barriers of the use of exoskeletons in small and medium-sized enterprises}

\section{Abstract}

Background. In the manufacturing industry, work-related musculoskeletal disorders (MSD) result in sick days and have substantial economic consequences for the enterprise and the national economy. Exoskeletons can support the body when handling heavy loads and enduring enforced postures. Exoskeletons are being piloted particularly in large companies in the automotive industry; however, exoskeletons have so far attracted little interest in small and medium-sized enterprises (SME) and their use has so far barely been scientifically examined. The aim of this work was to determine barriers to exoskeleton implementation and expectations for their use in the manufacturing sector. Method. Semi-structured guided interviews in six manufacturing companies were carried out and analyzed.

Results. In the enterprises a variety of activities up to the loading limits are carried out. Exoskeletons are generally expected to facilitate work and provide economic advantages. There are concerns with respect to their use due to cost factors, uncertain benefits and wearing discomfort. Particularly uncertainties about the effects of exoskeletons become evident.
Conclusion. The presented interview results are one step in an interdisciplinary process of further developing and implementing exoskeletons in the manufacturing industry. Concerns and unawareness of potential enterprises and users must be addressed, also to achieve a high user acceptance. Further studies that survey the identification of needs with better discriminatory power could provide additional insights.

Keywords Industrial exoskeleton - Work-related musculoskeletal disorders · Enduser - Manufacturing industry - Logistics ohne Rotation beinhalten. Es finden hohe Frequenzen des Arbeitsaufkommens, saisonal sowie auch während des Arbeitsalltags statt, es müssen repetitive und auch ausdauernde Positionen gehalten werden, und es kommt zu Überkopfarbeit. Die Unternehmen haben bereits arbeitserleichternde Maßnahmen getroffen, die technischer und organisatorischer Herkunft sind (Frage 1 in $\bullet$ Tab. 2).

Unterstützt werden sollen die obere, untere Extremität oder der Rücken, und zu unterstützende Tätigkeiten sind wiederholendes Heben, statische Haltungen und das Tragen. Vor allem sollen Exoskelette dann eine Unterstützung leisten, wenn technische Hilfsmittel ausgereizt sind (Frage 2 und 3 in • Tab. 2).

Der Begriff Exoskelett ruft sowohl positive, negative und neutrale Assoziationen hervor. Alle Nennungen sind unter Frage 5 in $\bullet$ Tab. 2 ersichtlich. Die meisten Antworten werden der positiven Kategorie zugeordnet. Neben der Prävention, Unterstützung durch Arbeitserleichterung, einer allgemein gesteigerten Ge- sundheit durch Schonung wird eine positiv beeinflusste Arbeitsmotivation erwartet. Negative Antworten beschreiben den Kosten-Nutzen-Aspekt, mit hohen Anschaffungskosten und Folgekosten, einer schlechten Nutzerakzeptanz aufgrund eines niedrigen Tragekomforts, und es wird ein geringer sowie ungewisser Nutzen mit dem Begriff Exoskelett verbunden. Allgemein wird ein Exoskelett von allen sechs Betrieben als ein externes Skelett, angesehen, welches sowohl aktiv als auch passiv bei Tätigkeiten unterstützt. 
Tab. 2 Übersicht der Fragen des Leitfadeninterviews, der gebildeten Kategorien, Subkategorien und zugehörige Beispiele aus den Antworten der Interviews

\begin{tabular}{|c|c|c|c|}
\hline Frage & Kategorie & Subkategorien & Beispiele \\
\hline \multirow{30}{*}{$\begin{array}{l}\text { 1. Welche schweren körperli- } \\
\text { chen Tätigkeiten, Bewegungs- } \\
\text { abläufe werden in Ihrem Be- } \\
\text { trieb durchgeführt? }\end{array}$} & \multirow{10}{*}{$\begin{array}{l}\text { Handhabung schwerer } \\
\text { Materialien }\end{array}$} & \multirow[t]{4}{*}{-} & Allg. Handling großer schwerer Materialien \\
\hline & & & Allg. repetitive manuelle schwere körperliche Tätigkeiten \\
\hline & & & Handling vibrierender, schwerer Werkzeuge \\
\hline & & & Schaufeln, Ausheben, Schweißen \\
\hline & & \multirow[t]{3}{*}{ Heben } & Schweres repetitives Heben \\
\hline & & & Überkopfheben, Absenken \\
\hline & & & Heben ohne Bücken \\
\hline & & Heben mit Rotation & Asymmetrisches Heben \\
\hline & & Tragen & Tragen schwerer Last \\
\hline & & Schieben & Schieben von schweren Lasten \\
\hline & \multirow[t]{2}{*}{ Belastungsspitzen } & \multirow[t]{2}{*}{-} & Hohes Arbeitsaufkommen saisonal \\
\hline & & & Treppensteigen \\
\hline & \multirow[t]{8}{*}{ Zwangshaltungen } & \multirow[t]{8}{*}{-} & Repetitiv, kurzweilig Zwangshaltungen \\
\hline & & & Zwangshaltungen längerer Dauer \\
\hline & & & Gebeugte Haltung \\
\hline & & & Kniende Haltung \\
\hline & & & Kauernde Haltung \\
\hline & & & Liegende Haltung \\
\hline & & & Überkopfarbeit \\
\hline & & & Steharbeit \\
\hline & \multirow{10}{*}{$\begin{array}{l}\text { Bereits getroffene } \\
\text { arbeitserleichternde } \\
\text { Maßnahmen }\end{array}$} & \multirow[t]{10}{*}{-} & Scherenhubtische \\
\hline & & & Stationswechsel \\
\hline & & & Aktives Exoskelett \\
\hline & & & Geneigte Regalebenen \\
\hline & & & Höhenverstellbares Regal \\
\hline & & & Wendomat \\
\hline & & & Robotik \\
\hline & & & Kräne \\
\hline & & & Lastenaufzüge \\
\hline & & & Rollentische \\
\hline \multirow{4}{*}{$\begin{array}{l}\text { 2. Welche Körperbereiche } \\
\text { erfordern Unterstützung? }\end{array}$} & Obere Extremität & - & Arme \\
\hline & Rücken & - & Wirbelsäule \\
\hline & Untere Extremität & - & Knie \\
\hline & Ganzer Körper & - & Muskel-Skelett System \\
\hline \multirow{7}{*}{$\begin{array}{l}\text { 3. Für welche Tätigkeiten } \\
\text { beabsichtigen Sie ggf. ein } \\
\text { Exoskelett einzusetzen? }\end{array}$} & \multirow[t]{4}{*}{ Umgang schwerer Last } & \multirow[t]{4}{*}{-} & Schaufeln \\
\hline & & & Tragen \\
\hline & & & Repetitives Heben \\
\hline & & & Asymmetrisches Heben \\
\hline & Zwangshaltungen & - & Statisches Halten \\
\hline & Vibrationsbelastung & - & Montagearbeit \\
\hline & $\begin{array}{l}\text { Wenn technische Hilfs- } \\
\text { mittel ausgereift sind }\end{array}$ & - & Arbeitsplatz ist maximal ergonomisch aufgebaut \\
\hline \multirow{4}{*}{$\begin{array}{l}\text { 4. Hatten Sie schon im berufli- } \\
\text { chen Kontext Erfahrungen mit } \\
\text { Exoskeletten? }\end{array}$} & \multirow[t]{2}{*}{ Ja } & \multirow[t]{2}{*}{-} & Ein querschnittsgelähmter ehemaliger MA kann damit gehen \\
\hline & & & Vorführung durch Hersteller \\
\hline & \multirow[t]{2}{*}{ Nein } & \multirow[t]{2}{*}{-} & Kontaktaufnahme zu Hersteller, hat sich verlaufen \\
\hline & & & Projekt mit Exo war geplant, hat sich verlaufen \\
\hline
\end{tabular}


Tab. 2 (Fortsetzung)

\begin{tabular}{|c|c|c|c|}
\hline Frage & Kategorie & Subkategorien & Beispiele \\
\hline \multirow{11}{*}{$\begin{array}{l}\text { 5. Was verbinden Sie mit dem } \\
\text { Begriff Exoskelett? }\end{array}$} & \multirow[t]{6}{*}{ Positiv } & Unterstützung & Arbeitserleichterung um die Hälfte \\
\hline & & \multirow[t]{2}{*}{ Gesundheit } & Schonung \\
\hline & & & Prävention \\
\hline & & Motivation & Mehr Freude bei der Arbeit \\
\hline & & Marketing & Zukunftsperspektive des Jobs \\
\hline & & Neugier & Ob es dadurch tatsächlich eine Entlastung gibt? \\
\hline & \multirow[t]{3}{*}{ Negativ } & $\begin{array}{l}\text { Kosten-Nutzen nicht } \\
\text { erfüllt }\end{array}$ & Hohe (Folge-)Kosten \\
\hline & & $\begin{array}{l}\text { Kein/geringer/ } \\
\text { ungewisser Nutzen }\end{array}$ & Krankenquote wird dadurch nicht verringert \\
\hline & & $\begin{array}{l}\text { Nutzerakzeptanz } \\
\text { niedrig }\end{array}$ & Diskomfort für Mitarbeiter \\
\hline & \multirow[t]{2}{*}{ Allgemein } & Externes Skelett & Aufgesetztes Skelett, Unterstützung von außen \\
\hline & & $\begin{array}{l}\text { Roboter/Maschine/ } \\
\text { Gerät }\end{array}$ & Passive, aktive Unterstützung (der Extremitäten) \\
\hline \multirow{15}{*}{$\begin{array}{l}\text { 6. Sammeln Sie Eigenschaften/ } \\
\text { Merkmale, die Ihnen zum } \\
\text { Thema Exoskelette einfallen }\end{array}$} & $\begin{array}{l}\text { Aktive und passive } \\
\text { Exoskelette }\end{array}$ & - & Es gibt angetriebene und nicht angetriebene \\
\hline & Einfluss auf Gesundheit & Prävention & Reduzierter Krankenstand durch Hilfsmittel \\
\hline & Unterstützung & Arbeitserleichterung & Entlastung für den MA \\
\hline & \multirow[t]{2}{*}{ Vorteil } & - & $\begin{array}{l}\text { Andere Hilfsmittel oft störend und nicht genutzt. Bei Exo größere } \\
\text { Bereitschaft, weil es direkt am Körper getragen wird }\end{array}$ \\
\hline & & $\begin{array}{l}\text { Ökonomisch, produk- } \\
\text { tiv }\end{array}$ & $\begin{array}{l}\text { Menschliche Ressourcen werden geschont, es kann schneller } \\
\text { gearbeitet werden }\end{array}$ \\
\hline & Innovatives Hilfsmittel & - & Neuartigkeit steigert Nutzerbereitschaft \\
\hline & Motivation Mitarbeiter & - & Mehr Freude bei der Arbeit \\
\hline & \multirow[t]{6}{*}{ Nutzerakzeptanz } & Hygiene & Schwitzen \\
\hline & & Diskomfort & Hohes Gewicht; es muss leicht sein \\
\hline & & Arbeitssicherheit & Es muss den Arbeitsschutzrichtlinien entsprechen \\
\hline & & Flexibilität & Es muss flexibel sein \\
\hline & & Optik & Es sieht abschreckend aus \\
\hline & & $\begin{array}{l}\text { Unwissenheit über } \\
\text { Nutzen, Vorteil }\end{array}$ & $\begin{array}{l}\text { MA meinen, sie brauchen es im Moment nicht; Wissen über Exo } \\
\text { fehlt }\end{array}$ \\
\hline & Langlebigkeit & - & Es sollte nicht sofort kaputt gehen \\
\hline & Kostenfaktor & - & Der hohe Kostenfaktor ist der große Haken an der Sache \\
\hline \multirow{7}{*}{$\begin{array}{l}\text { 7. Mit welchem Ziel/mit wel- } \\
\text { cher Absicht möchten Sie ein } \\
\text { Exoskelett in Ihrem Betrieb } \\
\text { einsetzen? }\end{array}$} & \multirow[t]{2}{*}{ Gesundheit } & $\begin{array}{l}\text { Krankenquote sen- } \\
\text { ken }\end{array}$ & Krankenquote sollte gesenkt werden \\
\hline & & Prävention & Spätere Schäden, im Alter, vermeiden \\
\hline & Unterstützung & $\begin{array}{l}\text { Erleichterung/ } \\
\text { Entlastung/Hilfe }\end{array}$ & Wir glauben daran, dass es den MA entlastet \\
\hline & \multirow[t]{3}{*}{ Vorteil } & Ökonomisch positiv & Geringerer Krankenstand ist positiv für Kosten \\
\hline & & Zukunftsorientiert & Attraktivitätssteigerung der Berufe \\
\hline & & Arbeitsakzeptanz & Mehr Freude bei der Arbeit, Motivationssteigerung \\
\hline & $\begin{array}{l}\text { Ergänzung zu unterstüt- } \\
\text { zenden Maßnahmen }\end{array}$ & - & $\begin{array}{l}\text { Ergänzung, wo wir mit technischen Mitteln am Ende angekom- } \\
\text { men sind }\end{array}$ \\
\hline \multirow{6}{*}{$\begin{array}{l}\text { 8. Welche Stärken hat Ihrer } \\
\text { Meinung nach der Einsatz von } \\
\text { Exoskeletten? }\end{array}$} & Unterstützung & Entlastung & Des Körpers, der Gelenke; Kraftunterstützung \\
\hline & Gesundheit & Prävention & Vorsorge für das Alter \\
\hline & $\begin{array}{l}\text { Innovativ/ } \\
\text { zukunftsorientiert }\end{array}$ & - & Neuartigkeit \\
\hline & Ökonomisch positiv & - & Schnelleres Arbeiten, weniger Krankheitstage \\
\hline & Motivation Mitarbeiter & - & Mehr Freude bei der Arbeit \\
\hline & Unwissenheit & Nutzen & Keine Erfahrung \\
\hline
\end{tabular}




\begin{tabular}{|c|c|c|c|}
\hline Frage & Kategorie & Subkategorien & Beispiele \\
\hline \multirow{12}{*}{$\begin{array}{l}\text { 9. Welche Schwächen hat Ihrer } \\
\text { Meinung nach der Einsatz von } \\
\text { Exoskeletten? }\end{array}$} & \multirow[t]{2}{*}{ Kosten } & - & Anschaffung lässt sich einfach nicht rechnen \\
\hline & & Folgekosten & Weitere Anschaffungen zum reinen Exo nötig \\
\hline & Nachteil & Ökonomisch & $\begin{array}{l}\text { Hilft nicht im operativen Geschäft, MA müssen weiter rotiert } \\
\text { werden zw. Stationen }\end{array}$ \\
\hline & \multirow[t]{6}{*}{ Nutzerakzeptanz } & $\begin{array}{l}\text { Bewegungs- } \\
\text { einschränkung }\end{array}$ & Arbeitsbehinderung, da keine spontanen Bewegungen möglich \\
\hline & & Flexibilität & Läuft noch nicht flüssig in Bewegungen, passt sich nicht an \\
\hline & & Gewicht & Hohes Eigengewicht \\
\hline & & Tragekomfort & Diskomfort \\
\hline & & Optik & Nicht ausgereift, sieht noch eigenartig aus \\
\hline & & Hygiene & Schwitzen \\
\hline & Entwicklungsstand & - & Noch nicht ausgereift \\
\hline & \multirow[t]{2}{*}{ Unwissenheit } & Nutzen & Keine Erfahrung \\
\hline & & Funktion & Keine Erfahrung \\
\hline \multirow{17}{*}{$\begin{array}{l}\text { 10. Was müssten Sie über } \\
\text { den Effekt von Exoskeletten } \\
\text { wissen, um deren Einsatz in } \\
\text { Ihrem Unternehmen zu befür- } \\
\text { worten? }\end{array}$} & Allgemein & Nutzen & Für MA, für Unternehmen \\
\hline & \multirow[t]{4}{*}{ Funktion } & Leistung & Wie viel Entlastung, ein Beispiel \\
\hline & & Effektivität & Vorteil durch Exo \\
\hline & & Einfluss MA & Schaden durch Exo für MA \\
\hline & & $\begin{array}{l}\text { Mechanische Funkti- } \\
\text { on }\end{array}$ & $\begin{array}{l}\text { Erklärung über mechanische Funktionsweise; dynamische/ } \\
\text { statische Anwendung }\end{array}$ \\
\hline & \multirow[t]{3}{*}{ Vorteile ökonomisch } & Förderung & - \\
\hline & & Marketing & Bringt es Vorteil für Ansehen des Berufes, des Unternehmens \\
\hline & & $\begin{array}{l}\text { Kosten-Nutzen- } \\
\text { Faktor }\end{array}$ & $\begin{array}{l}\text { Produktionssteigerung möglich durch Anschaffung; frühe Amorti- } \\
\text { sation }\end{array}$ \\
\hline & \multirow[t]{3}{*}{ Nachteile ökonomisch } & Kosten & Anschaffung \\
\hline & & Folgekosten & Versicherung, Pflege \\
\hline & & Wartungskosten & Reparaturen, Ersatzteile; Garantie \\
\hline & \multirow[t]{4}{*}{ Einsatzbereich } & $\begin{array}{l}\text { Anwendbarkeit auf } \\
\text { mehrere Nutzer }\end{array}$ & $\begin{array}{l}\text { Grundgerüst, das geteilt werden kann durch individuelle Erweite- } \\
\text { rungen }\end{array}$ \\
\hline & & Bedienbarkeit & Können selbst Änderungen vorgenommen werden \\
\hline & & Grenzen & Wo darf es nicht angewendet werden, draußen/drinnen \\
\hline & & Usability & Vielfältigkeit \\
\hline & Akzeptanz & - & Wie nehmen MA das Exo an? \\
\hline & Langzeitwirkung & - & Für MA, für Unternehmen \\
\hline $\begin{array}{l}\text { 11. Kennen Sie die Bestim- } \\
\text { mungen für den Einsatz von }\end{array}$ & Ja & - & - \\
\hline $\begin{array}{l}\text { Exoskeletten bzgl. Gesund- } \\
\text { heitsschutz und Arbeitssicher- } \\
\text { heit? }\end{array}$ & Nein & - & Nein \\
\hline Exo Exoskelett; MA Mitarbeiter*in & & & \\
\hline
\end{tabular}

$\mathrm{Zu}$ der Frage der vermuteten Eigenschaften und Merkmale von Exoskeletten wurden Merkmale, die die Nutzerakzep$\operatorname{tanz}$ des Exoskeletts betreffen, wie Hygiene und Flexibilität und auch Einflüsse auf die Gesundheit, wie ein reduzierter Krankenstand genannt (Frage 6 in - Tab. 2). Dem Exoskelett werden Vorteile gegenüber anderen Hilfsmitteln zugesprochen und auch ökonomische Vorteile durch Produktionssteigerungen. Es wird als arbeitsunterstützend für den Mitarbeiter und als motivationsfördernd gesehen. Exoskelette sollten sich durch Langlebigkeit auszeichnen. Des Weiteren wird Exoskeletten ein hoher Kostenfaktor zugesprochen.

Vor allem möchten Unternehmen durch den Einsatz eines Exoskeletts allgemein die Gesundheit fördern, Prävention betreiben und ebenso eine Erleichterung und Unterstützung der Arbeitsbelastung für ihre Mitarbeiter schaffen. Die Betriebe erwarten einen Vorteil, der sich ökonomisch zeigt, die Attraktivität des Berufes steigert und die Arbeitsakzeptanz erhöht. Es soll ergänzend zu bereits vorhandenen unterstützenden Maßnahmen ein Exoskelett zum Einsatz kommen (vgl. Frage 7 in $\bullet$ Tab. 2).

$\mathrm{Zu}$ den Stärken können zwei Unternehmen - aufgrund mangelnder Erfahrung und Kenntnisse - nicht antworten 
und verweisen auf die oben wiedergegebenen Absichten. Die vier übrigen Betriebe sehen die durch das Exoskelett geleistete Unterstützung als Stärke, ebenso wie die Prävention und Innovativität und bspw. einen ökonomischen Vorteil (Frage 8 in • Tab.2). Bei den Schwächen werden unterschiedliche Punkte angesprochen (Frage 9 in - Tab. 2). Es werden vor allem Schwächen im Bereich der Nutzerakzeptanz erwartet. Es wird eine Bewegungseinschränkung und Arbeitsbehinderung erwartet, ein hohes Eigengewicht und der Tragekomfort wird insgesamt als Schwäche angesehen. Auch wird ein hoher Kostenfaktor mit ökonomischem Nachteil genannt.

Bisher können die sechs befragten Unternehmen einem Einsatz noch nicht befürwortend entgegenstehen, da mangelnde Kenntnisse über das Thema Exoskelett bestehen. Es werden eine Aufklärung über die genaue Funktion eines Exoskeletts im Hinblick auf dessen mögliche Leistung und Effektivität erbeten und Wissen über den Einsatzbereich sowie Akzeptanz gefordert. Vor- und Nachteile über den Einsatz aus ökonomischer Sicht, wie Kosten-Nutzen-Faktoren, Förderungen aber auch Folgekosten und Wartungskosten werden erbeten (Frage 10 in - Tab. 2).

\section{Diskussion}

Es wurden anhand der Interviews Erwartungen an Exoskelette, Assoziationen und mögliche Barrieren von KMU der NSR aus den Bereichen der Logistik, dem Baugewerbe und der herstellenden Industrie aus den Leitfadeninterviews abgeleitet. Aus diesen Ergebnissen sollen mögliche Barrieren für eine erfolgreiche Implementierung von Exoskeletten adressiert werden.

In allen beteiligten Branchen werden unterschiedliche Tätigkeiten ausgeführt, ein Anwendungspotenzial von Exoskeletten wurde aus allen Interviews deutlich. So werden in KMU vielfältige Tätigkeiten und sich ändernde Arbeitsschritte durchgeführt (『Tab. 2). Exoskelette unterstützen bisher meist eine Tätigkeit, schränken jedoch eine andere Tätigkeit ein [15]. Ist die Tätigkeitspalette vielfältig und wechselnd, können sich so- mit Probleme in Anwendung und Nutzen von Exoskeletten ergeben. Die Akzeptanz dieser Unterstützung durch die Mitarbeiter ist demnach meist niedrig, da die wahrgenommene Einschränkung der Unterstützungswahrnehmung überwiegt $[1,15]$. Im Baugewerbe kommen zudem erschwerte Arbeitsplatzumfelder hinzu. Grenzen der Einsatzbedingungen und Sicherheitsfaktoren des Einsatzes eines Exoskeletts unter solchen Bedingungen sind bisher nicht erforscht [18].

Es ergeben sich hier Hinweise, dass eine generelle Unwissenheit über die Effektivität von Exoskeletten und deren möglichen auch ökonomischen Vorteilen besteht. Eine neue Technologie kann jedoch nur dann akzeptiert werden, wenn gesteigertes Wissen über den positiven Nutzen gegeben ist [12, 18, 25]. Ebenso muss ein Exoskelett zu den Arbeitsbedingungen, dem Arbeitsumfeld und den erwünschten Resultaten passend ausgewählt werden, da die subjektive Wahrnehmung um die Effektivität des Exoskeletts und die Akzeptanz für dessen Verwendung dadurch maßgeblich bestimmt werden [10]. Keinem der Ansprechpartner sind Bestimmungen für den Einsatz von Exoskeletten im Hinblick auf den Gesundheitsschutz und die Arbeitssicherheit geläufig (Frage 11 - Tab. 2). Nach diesen Bestimmungen müssen Gefährdungsbeurteilungen des Einsatzes am Arbeitsplatz, erforderliche Schutzmaßnahmen und Einweisungen erfolgen [11].

Eine Herausforderung bei der Datenaufnahme war die geringe Reaktion der KMU auf die Einladungen zur Teilnahme an den Interviews. Die geringe Rücklaufquote führte zu einem kleinen Erhebungskollektiv und könnte ein Zeichen für mangelnde Ressourcen und geringe Kenntnisse über den Nutzen von Exoskeletten in den Unternehmen sein. Es könnte auch ein mangelndes Interesse bei den angefragten Unternehmen vorliegen. Aus den hier gewonnenen Erkenntnissen kann abgeleitet werden, dass die Erwartungen an den Nutzen von Exoskeletten zum einen hoch sind, aber auch Unwissenheit und Skepsis gegenüber dem Nutzen bestehen. Werden diese Punkte nicht adressiert, kann keine erfolgreiche Implementierung erfolgen, da keine Akzeptanz vorherrscht. Dieses Problem ei- ner fehlenden Akzeptanz zeigt sich hier in den häufigen negativen Assoziationen eines Interviewpartners, in dessen KMU bereits ein aktives Exoskelett angeschafft wurde. Das aktive Exoskelett wird von den Mitarbeitern, durch fehlenden Tragekomfort, nicht angewandt, somit ist der Kosten-Nutzen-Aspekt nicht erfüllt.

In den Interviews fällt der oft genannte Kosten-Nutzen-Faktor auf, der für Entscheidungsträger des Unternehmens ein weiterer entscheidender Faktor zur Akzeptanz in den Unternehmen ist [9]. So scheiterte die Anschaffung von Exoskeletten bei zwei Interviewpartnern an den Anschaffungskosten, bei einem weiteren deckt der erwartete Nutzenfaktor des Einsatzes nicht die damit verbundenen Kosten. Die Unternehmen scheinen generell offen gegenüber einer Möglichkeit der weiteren Unterstützung ihrer Mitarbeiter. Dies wird durch die Antworten zu den Absichten des Einsatzes und die generellen Erwartungen, Prävention und Unterstützung deutlich.

Neben den Kosten könnten auch schlechte Erfahrungen mit technischen Maßnahmen wie festen Hebevorrichtungen ein Grund für mangelnde Akzeptanz sein, die sich aufgrund von Mehraufwand und Inflexibilität nicht durchsetzen konnten. Werden bereits angeschaffte technische Hilfsmittel nicht angewandt, wird zusammen mit mangelndem Wissen keine Akzeptanz für ein neues Hilfsmittel gewährleistet sein. Liegen wiederum positive Erfahrungen vor, wie in einem befragten KMU über ein höhenverstellbares Regal, welches die Arbeitsmotivation steigerte und den Krankenstand mindern konnte, zeigt sich deutlich ein positiver Effekt und eine hohe Nutzerakzeptanz gegenüber neuen Technologien. Insgesamt fällt trotz einiger beschriebener Bedenken auf, dass die Mehrzahl der Assoziationen mit dem Begriff Exoskelett, bei fehlenden Kenntnissen über die genaue Funktion, positiv ist. Es mangelt an Wissen über die Funktionsweise, den Nutzen und die Effekte von Exoskeletten, zum anderen scheinen die ökonomischen und sozialen Vorteile unbekannt. Dies führt dazu, dass generell eher Vermutungen über diese gemacht werden. Zu einigen Fragen wurden ähnliche Antworten durch die 
Interviewpartner gegeben. Dies könnte an einem teilweise fehlenden Wissen der Befragten und an einer zu geringen Trennschärfe der hier entwickelten Fragen liegen. Die hier generierten Ergebnisse können dennoch einen Überblick über den Stand des Wissens und der vorhandenen Erwartungen in den teilnehmenden Unternehmen schaffen und lassen Rückschlüsse auf das größere Kollektiv zu. Die befragten Personen haben alle Positionen in den Unternehmen inne, die nicht in den Nutzerkreis eines Exoskeletts zählen. An den Antworten zum potenziellen Exoskelettgebrauch wird das insofern deutlich, als wiederholend Aspekte eines ökonomischen Vorteils und Kosten-Nutzen-Faktoren genannt werden. Es ist anzunehmen, dass Nutzer eines Exoskeletts einen anderen Blickwinkel mitbringen würden [9]. Für die Interpretation dieser Ergebnisse muss dies beachtet werden, und es sollten in folgenden Interviews potenzielle Endnutzer einbezogen werden. Drei der Ansprechpartner ${ }^{\star}$ innen hatten schon Kontakt mit Exoskeletten, jedoch nicht im Rahmen eines Präventionsansatzes der betrieblichen Gesundheitsförderung, weshalb die Ergebnisse mit in die Auswertung aufgenommen wurden. Ein KMU nannte im Interview Erfahrungen mit dem Einsatz eines aktiven Exoskeletts. In dem Interview zeigten sich viele negative Assoziationen. Da die Interviews hier zunächst einen allgemeinen Überblick über den Wissensstand liefern sollten, wurde dieses Unternehmen trotzdem in die Studie eingeschlossen. In zukünftigen Interviews sollten die Interviewpartner weiter nach Erfahrung ohne und mit aktiven und passiven Exoskeletten differenziert werden und in welchen Unternehmensbereichen in welchem Umfang Exoskelette eingesetzt werden.

Barrieren bei der Implementierung von Exoskeletten sind ganz klar die Unwissenheit über Effekte und den Nutzen von Exoskeletten sowie die vielfältigen Tätigkeiten, die in KMU ausgeführt werden müssen. Potenzielle Unternehmen müssen aufgeklärt und ausführliche Tätigkeits- und Arbeitsplatzanalysen müssen generiert werden.

\section{Limitationen}

Es liegen folgende Limitationen vor:

- Es nahmen nur sechs Unternehmen teil. Es sind keine Schlüsse auf die Gesamtheit der KMU in NSR möglich.

- Die Unternehmen stammen aus unterschiedlichen Branchen, mit unterschiedlichen Erfahrungen im Bereich der Exoskelette.

- Verschiedene Fragen haben teilweise gleiche Antworten hervorgebracht, da das Wissen der Befragten nicht ausreichte und auf Vermutungen zurückgegriffen wurde.

- Eine quantitative Analyse ist durch die qualitative Datenerhebung nicht möglich.

\section{Ausblick}

Die erhobenen Daten zeigen, dass mit dem Einsatz von Exoskeletten beim Endnutzer unterschiedliche Assoziationen einhergehen. Die Akzeptanz des Endnutzers scheint eine der wichtigsten Stellschrauben in der Implementierung von Exoskeletten zu sein. Damit sich diese in KMU erhöht, muss Aufklärung betrieben und das Wissen über die nützlichen Effekte von Exoskeletten erweitert werden. Die in der Studie ermittelten Erwartungen der KMU sollten in der Implementierung Beachtung finden. So wünschen sich Unternehmen geringe Kosten, eine einfache Anwendbarkeit, einen wirtschaftlichen und produktiven Vorteil, die Möglichkeit der Prävention und eine Reduktion von Krankheitstagen sowie Motivation bei der Arbeit. Um eine erfolgreiche Einführung von Exoskeletten in KMU zu gewährleisten, muss der Entwicklungsprozess benutzerorientiert ablaufen [4]. Auch, da die Zuhilfenahme eines Exoskeletts häufig als Schwäche des Trägers angesehen und gedeutet wird [1]. Die positiven Effekte des Tragens eines Exoskeletts müssen dem Endnutzer erklärt, gezeigt und durch diesen gespürt werden. Nur mit Daten aus realen Anwendungsbereichen kann eine Anpassung an spezifische Arbeitsumgebungen und erfolgreiche Implementierung angegangen werden. Dieser Implementierungsprozess muss interdisziplinär sein, bestehend aus Nutzern und Interessenvertretern. Das zeigt sich auch in einem Verfahrensmodell, welches technische, kaufmännische und soziale Bereiche umfasst, um Exoskelette in der Industrie erfolgreich einzuführen [14]. Die in der vorliegenden Arbeit vorgestellten Interviewergebnisse sind ein Schritt im interdisziplinären Prozess der Implementierung und Weiterentwicklung von Exoskeletten unter Einbeziehung der Nutzer. Anhand dieser Ergebnisse können Barrieren, wie das fehlende Wissen über den Nutzen des Exoskelett-Einsatzes für Unternehmen der NSR aus der Logistik, dem Bauwesen und der herstellenden Industrie identifiziert und eliminiert werden.

\section{Fazit für die Praxis}

\section{- Erwartungen und Assoziationen mit dem Einsatz von Exoskeletten sind vielfältig. \\ - Mangelndes Wissen in Unternehmen bezüglich des Kosten-Nutzen-Fak- tors, der Anwendbarkeit, der Funk- tion und der Effektivität erschwert die Teilnahme von Unternehmen an Forschungsprojekten zur Implemen- tierung. \\ - Die Nutzerakzeptanz wird vielschich- tig beeinflusst. \\ - Exoskelette sollten in einem interdis- ziplinären Prozess, unter Einbindung der Endnutzer, implementiert und deren Nutzen in der Anwendung weiter erforscht werden.}

\section{Korrespondenzadresse}

Prof. Dr. Christoph Russmann

Fakultät für Ingenieurwissenschaften und Gesundheit, HAWK Hildesheim/Holzminden/ Göttingen

Göttingen, Deutschland

christoph.russmann@hawk.de

Förderung. Förderer: Das Projekt wird im Rahmen des Interreg-Projektes Exskallerate der EU gefördert. Förderzeitraum: 01/01/2020 bis 31/12/2023. Partner: Niederlande: InnovationQuarter, TNO Netherlands Organisation for Applied Scientific Research, University of Twente. Belgien: VUB (Vrije Universiteit Brussel), KUL (Katholieke Universiteit Leuven), POM - West Flanders. Deutschland: MoWiN - Regionalmanagement Nordhessen, HAWK. Großbritannien: Construction Scotland Innovation Centre, National Manufacturing Institute for Scotland (NMIS) at the University 
of Strathclyde. Schweden: University of Gävle. Dänmark: Aalborg University, Centre for Information Technology and Architecture (KADK). Internetpräsenz: https://northsearegion.eu/exskallerate/

Funding. Open Access funding enabled and organized by Projekt DEAL.

\section{Einhaltung ethischer Richtlinien}

Interessenkonflikt. H. Hoffmann, I. Pitz, B. Adomssent und C. Russmann geben an, dass kein Interessenkonflikt besteht.

Für diesen Beitrag wurden von den Autoren keine Studien an Menschen oder Tieren durchgeführt. Für die aufgeführten Studien gelten die jeweils dort angegebenen ethischen Richtlinien.

Open Access. Dieser Artikel wird unter der Creative Commons Namensnennung 4.0 International Lizenz veröffentlicht, welche die Nutzung, Vervielfältigung, Bearbeitung, Verbreitung und Wiedergabe in jeglichem Medium und Format erlaubt, sofern Sie den/die ursprünglichen Autor(en) und die Quelle ordnungsgemäß nennen, einen Link zur Creative Commons Lizenz beifügen und angeben, ob Änderungen vorgenommen wurden.

Die in diesem Artikel enthaltenen Bilder und sonstiges Drittmaterial unterliegen ebenfalls der genannten Creative Commons Lizenz, sofern sich aus der Abbildungslegende nichts anderes ergibt. Sofern das betreffende Material nicht unter der genannten Creative Commons Lizenz steht und die betreffende Handlung nicht nach gesetzlichen Vorschriften erlaubt ist, ist für die oben aufgeführten Weiterverwendungen des Materials die Einwilligung des jeweiligen Rechteinhabers einzuholen.

Weitere Details zur Lizenz entnehmen Sie bitte der Lizenzinformation auf http://creativecommons.org/ licenses/by/4.0/deed.de.

\section{Literatur}

1. Baltrusch SJ, van Dieën JH, van Bennekom CAM, Houdijk $H$ (2018) The effect of a passive trunk exoskeleton on functional performance in healthy individuals. Appl Ergon 72:94-106. https://doi. org/10.1016/j.apergo.2018.04.007

2. Baltrusch SJ, Houdijk H, van Dieën JH, de Kruif JTCM (2020) Passive trunk exoskeleton acceptability and effects on self-efficacy in employees with low-back pain: a mixed method approach. J Occup Rehabil. https://doi.org/10.1007/s10926-020-09891-1

3. BAuA/BMAS (2017) Sicherheit und Gesundheit bei der Arbeit - Berichtsjahr 2016. Unfallverhütungsbericht Arbeit (Bundesanstalt für Arbeitsschutz und Arbeitsmedizin)

4. Bornmann J, Schirrmeister B, Parth T, GonzalezVargas J (2020) Comprehensive development, implementation and evaluation of industrial exoskeletons. Curr Dir Biomed Eng. https://doi. org/10.1515/cdbme-2020-2001

5. Bosch T, van Eck J, Knitel K, de Looze M (2016) The effects of a passive exoskeleton on muscle activity, discomfort and endurance time in forward bending work. Appl Ergon 54:212-217. https:// doi.org/10.1016/j.apergo.2015.12.003
6. Bundesministerium für Arbeit und Soziales (2015) LasthandhabV - Verordnung über Sicherheit und Gesundheitsschutz bei der manuellen Handhabung von Lasten bei der Arbeit. https:// www.gesetze-im-internet.de/lasthandhabv/ BJNR184200996.html. Zugegriffen: 18. Jan. 2021

7. Bundesministeriums der Justiz und für Verbraucherschutz (1996) ArbSchG BGBI. I, S 1246

8. Mock CN, Nugent R, Kobusingye O, Smith KR (2017) Disease control priorities, 3. Aufl. Bd.7

9. Crea S, Beckerle P, de Looze M, de Pauw K, Grazi L, Kermavnar T, Masood J, O'Sullivan LW, Pacifico I, Rodriguez-Guerrero C, Vitiello N, Ristić-Durrant D, Veneman J (2021) Occupational exoskeletons: a roadmap toward large-scale adoption. Methodology and challenges of bringing exoskeletons to workplaces. Wearable Technol. https://doi.org/10 1017/wtc.2021.11

10. de Vries AW, de Looze (2019) The effect of arm support exoskeletons in realistic work activities: a review study. J Ergonomics 9(4):255. https://doi. org/10.35248/2165-7556.19.9.255

11. Deutsche Gesetzliche Unfallversicherung e.V. (2019) Einsatz von Exoskeletten an gewerblichen Arbeitsplätzen. https://publikationen.dguv.de/ widgets/pdf/download/article/3579. Zugegriffen: 28. Mai 2021

12. Elprama $S A$, Vannieuwenhuyze JTA, de Bock $S$, Vanderborght B, de Pauw K, Meeusen R, Jacobs A (2020) Social processes: what determines industrial workers' intention to use exoskeletons? Hum Factors 62(3):337-350. https://doi.org/10.1177/ 0018720819889534

13. European Agency for Safety and Health at Work, IKEl, Panteia (2019) Work-related musculoskeleta disorders: prevalence, costs and demographics in the EU (Publications Office)

14. Feldmann C, Kaupe V, Lucas M (2020) A procedural model for exoskeleton implementation in intralogistics (epubli)

15. Fox S, Aranko O, Heilala J, Vahala P (2020) Exoskeletons. JMTM 31(6):1261-1280. https://doi. org/10.1108/JMTM-01-2019-0023

16. Graham RB, Agnew MJ, Stevenson JM (2009) Effectiveness of an on-body lifting aid at reducing low back physical demands during an automotive assembly task: assessment of EMG response and user acceptability. Appl Ergon 40(5):936-942

17. Hensel R, Keil M (2018) Subjektive Evaluation industrieller Exoskelette im Rahmen von Feldstudien an ausgewählten Arbeitsplätzen. Z Arb Wiss 72(4):252-263. https://doi.org/10.1007/s41449018-0122-y

18. Howard J, Murashov VV, Lowe BD, Lu M-L (2020) Industrial exoskeletons: need for intervention effectiveness research. Am J Ind Med 63(3):201-208. https://doi.org/10.1002/ajim.23080

19. Huysamen K, Bosch T, de Looze M, Stadler KS, GrafE, O'Sullivan LW (2018) Evaluation of a passive exoskeleton for static upper limb activities. Appl Ergon 70:148-155. https://doi.org/10.1016/j. apergo.2018.02.009

20. Kim S, Moore A, Srinivasan D, Akanmu A, Barr A Harris-Adamson C, Rempel DM, Nussbaum MA (2019) Potential of Exoskeleton Technologies to Enhance Safety, Health, and Performance in Construction: Industry Perspectives and Future Research Directions. IIE Trans Occup 7(3):185-191. https://doi.org/10.1080/24725838.2018.1561557

21. Koopman AS, Kingma I, Faber GS, de Looze MP, van Dieën JH (2019) Effects of a passive exoskeleton on the mechanical loading of the low back in static holding tasks. J Biomech 83:97-103. https://doi. org/10.1016/j.jbiomech.2018.11.033
22. de Looze MP, Bosch T, Krause F, Stadler KS, O'Sullivan LW (2016) Exoskeletons for industrial application and their potential effects on physical work load. Ergonomics 59(5):671-681. https://doi. org/10.1080/00140139.2015.1081988

23. Maurice P, Čamernik J, Gorjan D, Schirrmeister B, Bornmann J, Tagliapietra L, Latella C, Pucci D, Fritzsche L, Ivaldi S, Babič J (2019) Evaluation of PAEXO, a novel passive exoskeleton for overhead work. Comput Methods Biomech Biomed Engin 22(sup1):S448-S450. https://doi.org/10.1080/ 10255842.2020.1714977

24. Mayring P (2015) Qualitative Inhaltsanalyse. Grundlagen und Techniken, 12. Aufl. Beltz, Weinheim, Basel

25. Moyon A, Poirson E, Petiot J-F (2019) Development of an acceptance model for occupational exoskeletons and application for a passive upper limb device. IIE Trans Occup 7(3-4):291-301. https:// doi.org/10.1080/24725838.2019.1662516

26. Nussbaum MA, Lowe BD, de Looze $M$, HarrisAdamson C, Smets M (2019) An introduction to the special issue on occupational exoskeletons. IIE Trans Occup 7(3-4):153-162. https://doi.org/10 1080/24725838.2019.1709695

27. Rashedi E, Kim S, Nussbaum MA, Agnew MJ (2014) Ergonomic evaluation of a wearable assistive device for overhead work. Ergonomics 57(12):1864-1874. https://doi.org/10.1080/ 00140139.2014 .952682

28. Schick R (2018) Einsatz von Exoskeletten in der Arbeitswelt. Zbl Arbeitsmed 68(5):266-269. https://doi.org/10.1007/s40664-018-0299-0

29. Schwerha DJ, McNamara N, Nussbaum MA, Kim S (2021) Adoption potential of occupational exoskeletons in diverse enterprises engaged in manufacturing tasks. Int J Ind Ergon 82:103103. https://doi.org/10.1016/j.ergon.2021.103103

30. Sinclair RC, Cunningham TR, Schulte PA (2013) A model for occupational safety and health intervention diffusion to small businesses. Am JInd Med 56(12):1442-1451. https://doi.org/10.1002/ ajim. 22263

31. Spada S, Ghibaudo L, Gilotta S, Gastaldi L, Cavatorta MP (2017) Investigation into the applicability of a passive upper-limb exoskeleton in automotive industry. Procedia Manuf 11:1255-1262. https:// doi.org/10.1016/j.promfg.2017.07.252

32. Steinhilber B, Luger T, Schwenkreis P, Middeldorf $S$, Bork H, Mann B, von Glinski A, Schildhauer TA, Weiler S, Schmauder M, Heinrich K, Winter G, Schnalke G, Frener P, Schick R, Wischniewski S Jäger M (2020) Einsatz von Exoskeletten im beruflichen Kontext zur Primär-, Sekundär-, und Tertiärprävention von arbeitsassoziierten muskuloskelettalen Beschwerden. Z Arb Wiss 74(3):227-246. https://doi.org/10.1007/s41449020-00226-7

33. Theurel J, Desbrosses K (2019) Occupational exoskeletons: overview of their benefits and limitations in preventing work-related musculoskeletal disorders. IIE Trans Occup 7(3-4):264-280. https:// doi.org/10.1080/24725838.2019.1638331

34. Thiehoff R (2002) Wirtschaftliche Bewertung der Arbeitsunfähigkeit durch muskuloskelettale Erkrankungen. Orthopade 31(10):949-956. https:// doi.org/10.1007/s00132-002-0350-9

35. Todorovic O, Constantinescu C, Popescu D (2018) Foundations for economic evaluation of exokeletons in manufacturing. Acta Tech Napocensis Appl Math Mech Eng 61(3 Special):221-230

36. Toxiri S, Näf MB, Lazzaroni M, Fernández J, Sposito M, Poliero T, Monica L, Anastasi S, Caldwell DG, Ortiz J (2019) Back-support exoskeletons for 
occupational use: an overview of technological advances and trends. IIETrans Occup 7(3-4):237-249. https://doi.org/10.1080/24725838.2019.1626303

37. Volkswagen AG 2020 (2020) Being tested: Audi production tests exoskeletons for overhead tasks.

https://www.volkswagenag.com/en/news/2019/ 12/audi_exoskeleton_2019.html.Zugegriffen: 4.

März 2021 